\title{
Atividade antioxidante e estimativa do teor de melanoidinas em cafés torrados comerciais
}

\author{
Antioxidant activity and estimation of melanoidin content in \\ commercial roasted coffee
}

\author{
Mariana Bortholazzi Almeida ${ }^{1}$; Marta de Toledo Benassi²*
}

\begin{abstract}
Resumo
O café contém vários componentes bioativos, em destaque os antioxidantes, cujo consumo tem sido correlacionado a redução da incidência de doenças crônicas e degenerativas. Dentre aproximadamente 100 espécies conhecidas do gênero Coffea as mais importantes economicamente no mercado internacional são Coffea arabica (café arábica) e Coffea canephora (café conilon), usualmente encontradas como blends em produtos comerciais. O café arábica apresenta melhor qualidade sensorial. Os compostos aos quais é atribuída a atividade antioxidante (AA) das bebidas de café incluem a classe de fenóis (destaque para o 5-ACQ) e cafeína, que têm sido mais usualmente estudados, e melanoidinas, produzidas no processo de torra, para as quais dados na literatura são escassos. Diferentes métodos são utilizados para caracterizar a capacidade antioxidante de alimentos, mas não existe uma metodologia eficiente e universal de medida. Nesse trabalho, cafés torrados e moídos nacionais comercializados com diferentes denominações (37 amostras), foram analisados quanto a concentração de melanoidinas, por espectrofotometria, e atividade antioxidante pelo método ABTS e de fenólicos totais (Folin- Ciocalteau). Todos os produtos avaliados apresentaram expressiva atividade antioxidante, mas observaram-se diferenças na estimativa do teor de melanoidinas, indicando processos de torra diferenciados. Considerando-se as diferentes categorias, cafés tipo Gourmet se destacaram pelos menores valores de AA, demonstrando que além do grau de torra a espécie de café empregada influenciava a funcionalidade do produto.
\end{abstract}

Palavras-chave: Coffea arabica, Coffea canephora, fenóis totais, índice de escurecimento, ABTS

\begin{abstract}
Coffee contains several bioactive compounds, highlighting the antioxidants, whose consumption has been correlated with reduced incidence of chronic and degenerative diseases. Among 100 known species of the genus Coffea the most important economically in the international trade are Coffea arabica (arabica coffee) and Coffea canephora (robusta coffee), usually found as blends in commercial products. Arabica coffees present a higher sensory quality. The components associated with the antioxidant activity (AA) of coffee beverages included the class of phenolic compounds (especially 5-CQA) and caffeine, which have been most commonly studied, and melanoidins produced in the roasting process, for whom no data are available in the literature. Different methods are used to characterize the antioxidant capacity of foods, but there is no efficient and universal methodology of measurement. In this paper, roast and ground Brazilian coffees, commercialized under different designations (37 samples) were analyzed for the level of melanoidins, by spectrophotometry, and the antioxidant activity, by ABTS and total phenolics (FolinCiocalteau). All the products evaluated showed significant antioxidant activity. Differences observed in the estimation of the content of melanoidins indicated different roasting processes. Considering the
\end{abstract}

1 Bolsista de Iniciação Científica, Universidade Estadual de Londrina, UEL, Londrina, PR. E-mail: maribortholazzi@hotmail.com

2 Docente, Dr $^{\mathrm{a}}$. do Dept $^{\mathrm{o}}$ de Ciência e Tecnologia de Alimentos, Universidade Estadual de Londrina, UEL, Londrina, PR. E-mail: martatb@uel.br

* Autor para correspondência

Recebido para publicação 16/09/2010 Aprovado em 22/08/2011 
different categories, the Gourmet coffees were characterized for lower values of AA, indicating that besides the degree of roasting, the coffee species used influenced the functionality of the product.

Key words: Coffea arabica, Coffea canephora, total phenolics, browning index, ABTS

\section{Introdução}

O café, além de ser uma bebida altamente aceita e consumida em todo o mundo, é rico em compostos que possuem potenciais efeitos benéficos para a saúde humana, destacando-se pela expressiva atividade antioxidante. Compostos oxidantes são produzidos pelo metabolismo normal do organismo e, se não controlados, podem provocar maior incidência de doenças crônicas e degenerativas. Estudos clínicos e epidemiológicos têm mostrado evidências de redução da incidência dessas doenças em populações cujas dietas são altas na ingestão de alimentos ricos em compostos fenólicos (HIGDON; FREI, 2006).

O café é uma rica fonte de fenólicos, principalmente ácidos clorogênicos, destacando-se o isômero ácido 5-cafeoilquínico (5-ACQ), e seus produtos de degradação (ácidos caféico, ferúlico e cumárico). A atividade antioxidante (AA) no café tem sido correlacionada tanto a presença de compostos fenólicos como ácido clorogênico, quanto a vários outros compostos nitrogenados como trigonelina e cafeína, e melanoidinas. No entanto, variações na composição dos grãos utilizados e processamento afetam essa característica (HIGDON; FREI, 2006; PARRAS et al., 2007; VIGNOLI; BASSOLI; BENASSI, 2011).

As espécies de café mais comercializadas mundialmente são Coffea arabica e Coffea canephora (conilon). Cafés arábica apresentam maior valor comercial e qualidade sensorial, entretanto, o conilon possui um maior teor de sólidos solúveis. Assim, usualmente são empregados misturas de cafés de diferentes qualidade e espécies (blends) para formulação de produtos comerciais. São descritos para cafés torrados e moídos três categorias: Tradicional e Superior (podem ser blends com conilon) e Gourmet (somente arábica).
Enquanto as designações Tradicional, Superior e Gourmet dizem respeito à qualidade, os termos clara, média e escura são empregados para indicar o grau de torra (ABIC, 2011; BRASIL, 2010).

Diferentes técnicas são utilizadas para caracterizar a AA, entretanto, não há nenhuma metodologia universal pelo qual ela possa ser medida precisa e quantitativamente. A AA de café vem sendo avaliada por diferentes métodos, destacando-se o ABTS (SÁNCHEZ-GONZALEZ; JIMÉNEZ-ESCRIG; SAURA-CALIXTO, 2005). $\mathrm{O}$ método de Folin determina o teor de polifenóis totais e apresenta como mecanismo básico uma reação de óxido-redução, permitindo avaliar a AA (PRIOR; WU; SCHAICH, 2005).

Durante o processo de torra ocorre a degradação dos compostos fenólicos e outros componentes termólábeis (FARAH et al., 2005). Apesar do decréscimo no teor de polifenóis, recentemente têm sido mostrado que ácido quínico e caféico, componentes dos ácidos clorogênicos, são quimicamente incorporados às melonoidinas durante este processo (BEKEDAM et al., 2008). Entre os componentes com potencial atividade antioxidante e que são pouco afetados pelo processo de torra, destaca-se a cafeína (SHI; DALAL; JAIN, 1991).

Em trabalho anterior, Souza et al. (2010) analisaram alguns compostos bioativos e/ou de interesse na qualidade (5-ACQ, cafeína, trigonelina, ácido nicotínico, caveol e cafestol) em cafés comerciais de mercado com diferentes denominações. Observou-se, apesar da pequena variação na torra, grande diversidade na composição, indicando prováveis diferenças nas matérias primas e espécies de café utilizadas nos blends. É provável que essa heterogeneidade na composição leve também a diversidade na AA. 
Dessa forma, o objetivo do trabalho foi avaliar a atividade desses cafés torrados e moídos comerciais através das metodologias de ABTS e FolinCiocalteau, de maneira a relacionar as diferenças na composição com o impacto na AA. Para completar a informação, avaliou-se, em paralelo, os teores de melanoidinas, compostos com grande potencial AA e que não haviam sido analisados no estudo anterior.

\section{Material e Métodos}

Foram estudadas 37 amostras de cafés torrados e moídos comerciais, de 23 marcas, pertencentes a 13 fabricantes. As amostras, acondicionadas em sacos plásticos, foram armazenadas em câmara fria a 10 ${ }^{\circ} \mathrm{C}$ até análise. As determinações foram realizadas em duplicata, empregando-se um delineamento inteiramente ao acaso.

\section{Metodologia ABTS (TEAC)}

A capacidade antioxidante das soluções de café frente ao radical livre $\mathrm{ABTS}+\bullet$ foi avaliada de acordo com Sánchez-Gonzalez, JiménezEscrig e Saura-Calixto (2005). O cátion ABTS+• foi produzido reagindo $7 \mathrm{mM}$ da solução estoque ABTS com 2,45 mM de persulfato de potássio. A mistura permaneceu em frasco escuro em temperatura ambiente por 12-16 horas antes do uso. Esta solução foi então diluída com tampão salino fosfato $(\mathrm{pH} 7,4)$ para uma absorvância de 0,7 a $730 \mathrm{~nm}$. Adicionou-se amostra ou padrão Trolox $(10 \mu \mathrm{L})$ a solução ABTS+• diluída $(4 \mathrm{~mL})$ e, após 6 minutos de reação, as leituras a $730 \mathrm{~nm}$ foram realizadas. Soluções de etanol com concentrações conhecidas de Trolox foram usadas para calibração $(2,5 ; 5,0 ; 7,5 ; 12,5$ e $20,0 \mu \mathrm{Mol} / \mathrm{L})$. As amostras foram preparadas na concentração de $15 \mathrm{mg} \cdot \mathrm{mL}^{-1}$, resultando num filtrado com concentração de 4,33 mg. $\mathrm{mL}^{-1}$ de sólidos solúveis. Os resultados foram expressos como capacidade antioxidante equivalente ao Trolox (TEAC) (g de Trolox.100 g $\left.\operatorname{amostra}^{-1}\right)$.

\section{Metodologia de Folin-Ciocalteau}

A quantificação de compostos fenólicos totais foi realizada por Folin-Ciocalteau, método que envolve a redução do reagente pelos compostos fenólicos das amostras com concomitante formação de um complexo azul cuja intensidade aumenta linearmente a $760 \mathrm{~nm}$ (SWAIN; HILLIS, 1959). O teor total de fenóis de cada extrato foi quantificado por meio de uma curva padrão preparada com ácido gálico, e expresso como equivalentes de ácido gálico (GAE). Para a reação colorimétrica, uma alíquota de $0,5 \mathrm{~mL}$ da solução metanólica de extrato (concentração $0,5 \mathrm{mg}$ sólidos. $\mathrm{mL}^{-1}$ ) foi adicionada de $2,5 \mathrm{~mL}$ de solução aquosa do reativo Folin-Ciocalteau a $10 \%$ e $2,0 \mathrm{~mL}$ de carbonato de sódio a 7,5\%. A mistura foi incubada por 5 minutos em banho-maria a $50{ }^{\circ} \mathrm{C}$ e, posteriormente, a absorvância foi medida usando-se um branco como referência. Os resultados foram expressos como $\mathrm{g}$ ácido gálico. $100 \mathrm{~g}$ amostra $^{-1}$.

\section{Determinação de melanoidinas}

$\mathrm{O}$ teor de melanoidinas é tradicionalmente avaliado pela medida da absorvância a $420 \mathrm{~nm}$. A leitura foi realizada em um extrato filtrado obtido a partir de $5 \mathrm{~g}$ de café torrado e $50 \mathrm{~mL}$ de água destilada (WEN-JEY; BOR-SEN; PIN-DER, 2005).

\section{Resultados e Discussão}

Os teores de atividade antioxidante encontrados nos cafés comerciais analisados estão apresentados na Tabelas 1 a 5, separados de acordo com as diferentes denominações dos produtos. As marcas estão designadas por letras (A a W), e as denominações pelos códigos $\mathrm{T}$ (Tradicional), F (Forte), EF (Extra Forte), G (Gourmet), P (Premium), A (Aralto), EX (Exportação) e ES (Espresso). Apesar da variabilidade observada, todas as amostras apresentaram expressiva AA. 
Tabela 1. Melanoidinas e atividade antioxidante em cafés de denominação Tradicional $(\mathrm{T})$.

\begin{tabular}{lccc}
\hline Amostra & $\begin{array}{c}\text { ABTS (g de Trolox. } \\
\mathbf{1 0 0} \mathbf{g ~ a m o s t r a}^{-1} \mathbf{~}\end{array}$ & $\begin{array}{c}\text { Fenólicos Totais } \mathbf{( g} \text { ac. gálico. } \\
\left.\mathbf{1 0 0} \mathbf{~} \mathbf{~ a m o s t r a}^{-1}\right)\end{array}$ & $\begin{array}{c}\text { Melanoidinas } \\
(\mathbf{A b s} \mathbf{4 2 0} \mathbf{~ n m})\end{array}$ \\
\hline CT & $4,78 \pm 0,24$ & $3,08 \pm 0,10$ & $0,396 \pm 0,004$ \\
ET & $4,42 \pm 0,23$ & $2,79 \pm 0,48$ & $0,461 \pm 0,011$ \\
GT & $2,96 \pm 0,23$ & $2,66 \pm 0,16$ & $0,505 \pm 0,001$ \\
HT & $5,30 \pm 0,35$ & $3,55 \pm 0,04$ & $0,419 \pm 0,013$ \\
KT & $4,30 \pm 0,35$ & $1,77 \pm 0,10$ & $0,413 \pm 0,005$ \\
LT & $6,76 \pm 0,22$ & $3,06 \pm 0,09$ & $0,561 \pm 0,001$ \\
MT & $5,97 \pm 0,70$ & $1,91 \pm 0,04$ & $0,352 \pm 0,003$ \\
QT & $4,60 \pm 0,17$ & $2,33 \pm 0,01$ & $0,641 \pm 0,018$ \\
VT & $5,45 \pm 0,14$ & $2,96 \pm 0,10$ & $0,429 \pm 0,006$ \\
\hline
\end{tabular}

*Média de duas repetições \pm desvio padrão (DP).

Tabela 2. Melanoidinas e atividade antioxidante em cafés de denominação Forte (F).

\begin{tabular}{|c|c|c|c|}
\hline$\overline{\text { Amostra }}$ & $\begin{array}{c}\text { ABTS (g de Trolox.100 } \\
\text { g amostra } \\
\end{array}$ & $\begin{array}{l}\text { Fenólicos Totais (g ac. } \\
{\text { gálico. } 100 \mathrm{~g} \text { amostra }}^{-1} \text { ) }\end{array}$ & $\begin{array}{l}\text { Melanoidinas } \\
(\text { Abs } 420 \mathrm{~nm})\end{array}$ \\
\hline$\overline{\mathrm{EF}}$ & $5,15 \pm 0,22$ & $3,21 \pm 0,10$ & $0,488 \pm 0,004$ \\
\hline $\mathrm{NF}$ & $4,63 \pm 0,33$ & $3,27 \pm 0,04$ & $0,425 \pm 0,001$ \\
\hline $\mathrm{PF}$ & $5,18 \pm 0,06$ & $2,89 \pm 0,01$ & $0,432 \pm 0,013$ \\
\hline $\mathrm{TF}$ & $3,48 \pm 0,21$ & $1,77 \pm 0,08$ & $0,359 \pm 0,002$ \\
\hline
\end{tabular}

*Média de duas repetições \pm desvio padrão (DP).

Tabela 3. Melanoidinas e atividade antioxidante em cafés de denominação Extra Forte (EF).

\begin{tabular}{|c|c|c|c|}
\hline Amostra & 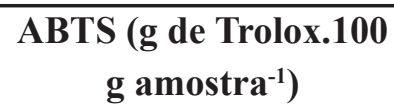 & $\begin{array}{c}\text { Fenólicos Totais (g ac. gálico. } \\
\left.100 \mathrm{~g} \mathrm{amostra}^{-1}\right)\end{array}$ & $\begin{array}{l}\text { Melanoidinas } \\
(\text { Abs } 420 \mathrm{~nm})\end{array}$ \\
\hline$\overline{\mathrm{AEF}}$ & $4,47 \pm 0,07$ & $3,20 \pm 0,09$ & $0,406 \pm 0,004$ \\
\hline DEF & $4,87 \pm 0,60$ & $3,33 \pm 0,12$ & $0,459 \pm 0,006$ \\
\hline EEF & $4,06 \pm 0,01$ & $2,18 \pm 0,10$ & $0,466 \pm 0,002$ \\
\hline FEF & $5,53 \pm 0,60$ & $1,68 \pm 0,06$ & $0,358 \pm 0,034$ \\
\hline HEF & $6,14 \pm 0,41$ & $3,63 \pm 0,09$ & $0,690 \pm 0,013$ \\
\hline IEF & $3,42 \pm 0,38$ & $2,73 \pm 0,04$ & $0,358 \pm 0,006$ \\
\hline JEF & $4,91 \pm 0,07$ & $1,62 \pm 0,01$ & $0,349 \pm 0,006$ \\
\hline OEF & $5,22 \pm 0,03$ & $2,44 \pm 0,08$ & $0,369 \pm 0,001$ \\
\hline QEF & $4,82 \pm 0,57$ & $2,13 \pm 0,20$ & $0,371 \pm 0,006$ \\
\hline REF & $3,80 \pm 0,11$ & $2,21 \pm 0,07$ & $0,378 \pm 0,018$ \\
\hline SEF & $3,56 \pm 0,30$ & $1,86 \pm 0,12$ & $0,508 \pm 0,019$ \\
\hline SEF class & $3,02 \pm 0,28$ & $2,47 \pm 0,01$ & $0,379 \pm 0,004$ \\
\hline VEF & $6,31 \pm 0,09$ & $1,78 \pm 0,09$ & $0,447 \pm 0,009$ \\
\hline
\end{tabular}

*Média de duas repetições \pm desvio padrão (DP). 
Tabela 4. Melanoidinas e atividade antioxidante em cafés de denominação Premium (P), Aralto (A), Exportação (EX) e Espresso (ES).

\begin{tabular}{lccc}
\hline Amostra & $\begin{array}{c}\text { ABTS (g de Trolox.100 } \\
\text { g amostra }^{-1} \text { ) }\end{array}$ & $\begin{array}{c}\text { Fenólicos Totais (g ac. } \\
\left.\text { gálico. } \mathbf{~ 1 0 0 ~} \mathbf{g} \text { amostra }^{-1}\right)\end{array}$ & $\begin{array}{c}\text { Melanoidinas } \\
(\mathbf{A b s ~ 4 2 0 ~} \mathbf{~ m})\end{array}$ \\
\hline BP & $5,53 \pm 0,22$ & $2,46 \pm 0,07$ & $0,393 \pm 0,015$ \\
HP & $5,01 \pm 0,01$ & $2,90 \pm 0,10$ & $0,423 \pm 0,001$ \\
DA & $3,94 \pm 0,45$ & $2,81 \pm 0,03$ & $0,316 \pm 0,001$ \\
DEX & $6,07 \pm 0,12$ & $4,11 \pm 0,09$ & $0,606 \pm 0,012$ \\
WES & $3,80 \pm 0,44$ & $1,58 \pm 0,08$ & $0,391 \pm 0,028$ \\
\hline
\end{tabular}

*Média de duas repetições \pm desvio padrão (DP).

Tabela 5. Melanoidinas e atividade antioxidante em cafés de denominação Gourmet (G).

\begin{tabular}{|c|c|c|c|}
\hline Amostra & $\begin{array}{c}\text { ABTS (g de Trolox.100 } \\
\left.\text { g amostra }^{-1}\right)\end{array}$ & $\begin{array}{l}\text { Fenólicos Totais (g ac. } \\
\text { gálico.100 } \mathrm{g} \text { amostra }^{-1} \text { ) }\end{array}$ & $\begin{array}{l}\text { Melanoidinas } \\
(\text { Abs } 420 \mathrm{~nm})\end{array}$ \\
\hline$\overline{\mathrm{BG}}$ & $3,21 \pm 0,06$ & $1,58 \pm 0,08$ & $0,422 \pm 0,019$ \\
\hline $\mathrm{CG}$ & $4,15 \pm 0,53$ & $2,16 \pm 0,07$ & $0,299 \pm 0,008$ \\
\hline FG & $3,87 \pm 0,04$ & $1,86 \pm 0,09$ & $0,310 \pm 0,004$ \\
\hline IG & $2,28 \pm 0,21$ & $1,96 \pm 0,06$ & $0,291 \pm 0,001$ \\
\hline UG & $3,90 \pm 0,01$ & $1,83 \pm 0,15$ & $0,334 \pm 0,015$ \\
\hline
\end{tabular}

*Média de duas repetições \pm desvio padrão (DP).

Os valores de melanoidinas tiveram grande variabilidade, na faixa de 0,291 a 0,690 em absorvância medida a 420nm, indicando probabilidade de terem ocorrido diferenças expressivas nos processos de torra empregados. O aumento no teor de melanoidinas se dá com a evolução do grau de torra, devido ao desenvolvimento da reação de Maillard. De acordo com Nicoli, Anese e Parpinel (1997) e Daglia et al. (2004) as melanoidinas contribuem em aproximadamente com $25 \%$ da matéria seca, sendo assim, um dos principais componentes da bebida do café torrado. Não se observou relação direta entre a presença de melanoidinas, indicativa de maior grau de torra, e as denominações empregadas para os cafés (Tradicional, Forte ou Extra-forte). Esse comportamento já havia sido descrito por Souza et al. (2010), que não relatou correlação entre a denominação comercial indicativa de torra com a degradação de compostos termolábeis (5-ACQ e trigonelina).

Observou-se, no entanto, que o maior valor de melanoidinas foi obtido para uma amostra ExtraForte (HEF), e os cafés Gourmet apresentaram alguns dos teores mais baixos (menor valor foi da amostra IG), coincidentes com um grau de torra mais leve usualmente empregado para esse tipo de produto (Tabelas 1 a 5 ).

Constatou-se também grande variabilidade na AA considerando os dois métodos de medida. Os valores de ABTS variaram na faixa de 2,96 (GT) a 6,76 (LT) g de Trolox. $100 \mathrm{~g}_{\text {amostra }^{-1}}$, os de fenóis totais, de 1,58(WES) a 4,11 (DEX) g ac. gálico. $100 \mathrm{~g}$ amostra $^{-1}$. Cabe destacar o café DEX, que apresentou valores elevados de AA para os dois métodos, e os cafés do tipo Gourmet, com valores de AA inferiores as outras denominações (Tabelas 1 a 5$)$.

Vignoli, Bassoli e Benassi (2011), estudando 
cafés solúveis com diferentes graus de torra, espécies e métodos de extração, encontraram alta correlação entre AA medida por Folin e ABTS. No geral, observou-se que amostras que apresentaram elevados teores de fenóis totais também tiveram alta AA estimada pelo ABTS, no entanto não se observou correlação direta entre os dois métodos em todos os casos. Valores de Folin na faixa de 3 a 3,2 $\mathrm{g}$ ácido gálico. $100 \mathrm{~g}$ amostra $^{-1}$ corresponderam a AA, estimada por ABTS, bastante diferenciada: 4,47 (amostra EF), 5,45 (VT) e 6,76 (LT) g Trolox. $100 \mathrm{~g} \mathrm{amostra}^{-1}$ (Tabelas 1 a 5).

Esses resultados podem ser melhor avaliados considerando também os dados de composição em termos de 5-ACQ e cafeína descritos por Souza et al. (2010) para as mesmas amostras, e apresentados resumidamente na forma de valores médios para cada categoria na Tabela 6 .

Tabela 6. Teores de 5-ACQ e cafeína (g.100 g de amostra $^{-1}$ ) dos cafés de mercado (adaptado de SOUZA et al., 2010).

\begin{tabular}{lcc}
\hline Amostra & 5-ACQ & Cafeína \\
\hline Tradicional & $0,33 \pm 0,18$ & $1,50 \pm 0,25$ \\
Forte & $0,37 \pm 0,11$ & $1,51 \pm 0,26$ \\
Extra-Forte & $0,35 \pm 0,06$ & $1,56 \pm 0,19$ \\
Gourmet & $0,76 \pm 0,36$ & $1,19 \pm 0,12$ \\
Premium & $0,42 \pm 0,05$ & $1,47 \pm 0,18$ \\
DA & 0,60 & 1,09 \\
DEX & 0,57 & 1,43 \\
WES & 0,46 & 1,73 \\
\hline
\end{tabular}

Média \pm desvio padrão de 9 (T), 4 (F), 13 (EF), 5 (G) e 2 amostras (P). As denominações A, EX e EP contam com apenas uma amostra não tendo desvio.

Inicialmente, é interessante comparar a relação entre o teor de 5-ACQ, bastante representativo do comportamento dos compostos fenólicos livres, e o resultado de fenóis totais obtido por Folin (Tabelas 1 a 6). Quanto mais elevada a intensidade da torra no café, maior a degradação dos ácidos clorogênicos (representados, neste caso, pelo 5 ACQ). Cafés Gourmet considerados menos torrados, levandose em conta a estimativa de melanoidinas (Tabela 5), apresentam maior teor de 5-ACQ (Tabela 6). A literatura descreve que, aproximadamente metade dos ácidos clorogênicos degradados no processo de torra podem ser encontrados como pigmentos, na forma de ácido quínico livre e compostos fenólicos de baixa massa molecular (TRUGO; DE MARIA; WERNECK, 1991), sendo mais recentemente descrita a participação desses compostos na estrutura das melanoidinas do café (NUNES; COIMBRA, 2001; BEKEDAM; SCHOLS; VAN BOEKEL, 2006; BEKEDAM et al., 2008). Valores próximos de fenóis totais $(2,68$ e 2,81 g de ac. gálico. $100 \mathrm{~g}$ de amostra $^{-1}$ ) foram observados para amostras com perfil de composição diferenciado: café DA, com 0,60 g 5-ACQ.100 g ${ }^{-1}$ e absorvância de 0,316, e cafés Premium (valores médios de BP e HP), com 0,42 g 5-ACQ.100g-1 e absorvância de 0,401 (Tabelas 4 e 6). Dessa forma, observa-se que resultados de fenóis totais por Folin provavelmente expressam não apenas os compostos livres, mas também compostos associados a estrutura da melanoidina, avaliando de forma global a contribuição de componentes fenólicos para a AA.

Vignoli (2009), estudando compostos de café associados a AA, relatou que, além dos ácidos clorogênicos, a melanoidina também apresentou uma expressiva AA medida por ABTS. Cafés Gourmet, que tem níveis mais altos de 5-ACQ em função do menor grau de torra, apresentam um baixo teor de cafeína, associado ao uso exclusivo de café arábica (ABIC, 2011; SOUZA et al., 2010), e AA por ABTS correspondentemente mais baixa (Tabelas 5 e 6). Vignoli (2009) descreveu maior AA, medida por diferentes metodologias, para café conilon em comparação com o arábica independentemente do grau de torra dos cafés torrados e moídos estudados.

Dessa forma, observou-se que a AA dos cafés foi resultado do balanço do conteúdo de compostos antioxidantes que podem variar apenas com a espécie (cafeína), processo de torra (melanoidinas) 
ou ambos (5-ACQ).

\section{Conclusões}

Todos os produtos avaliados apresentaram expressiva atividade antioxidante. Observaram-se diferenças na estimativa do teor de melanoidinas, indicando processos de torra diferenciados para os cafés estudados. Considerando-se as diferentes categorias de café, produtos do tipo Gourmet se destacaram pelos menores valores de AA, demonstrando que além do grau de torra a espécie de café empregada influenciava a funcionalidade da bebida.

\section{Agradecimentos}

Agradecimentos ao $\mathrm{CNPq}$ pela concessão das bolsas de IC e produtividade.

\section{Referências}

ABIC programa de qualidade do café. 2011. Disponível em: $\quad<$ http://www.abic.com.br/gar_qualidade.html.>. Acesso em: 18 jul. 2011.

BEKEDAM, E. K.; SCHOLS, H. A.; VAN BOEKEL, M. A. J. S. High molecular weight melanoidins from coffee brew. Journal of Agricultural and Food Chemistry, Washington, v. 54, n. 20, p. 7658-7666, 2006.

BEKEDAM, E. K.; SCHOLS, H. A.; VAN BOEKEL, M. A. J. S.; SMIT, G. Incorporation of chlorogenic acids in coffee brew melanoidins. Journal of Agricultural and Food Chemistry, Washington, v. 56, n. 6, p. 2055-2063, 2008.

BRASIL. Instrução Normativa $\mathrm{n}^{\circ} 16$, de 24 de maio de 2010, do Ministério da Agricultura, Pecuária e Abastecimento. "Regulamento Técnico para o Café Torrado em Grão e do Café Torrado e Moído". Diário Oficial [da] União, Brasília, 25 maio 2010. Seção 1. Disponível em: <http://extranet.agricultura.gov.br/ sislegis/action/detalhaAto.do?method=consultarLegislac aoFederal.>. Acesso em: 18 jul. 2011.

DAGLIA, M.; RACCHI, M.; PAPETTI, A.; LANNI, C.; GOVONI, S.; GAZZANI, G. In vitro and ex vivo antihydroxil radical activity of green and roasted coffee. Journal of Agricultural and Food Chemistry, Washington, v. 52, n. 6, p. 1700-1704, 2004.
FARAH, A.; DE PAULIS, T.; TRUGO, L. C.; MARTIN, P. R. Effect of roasting on the formation of chlorogenic acids lactones. Journal of Agricultural and Food Chemistry, Washington, v. 53, n. 5, p. 1505-1513, 2005.

HIGDON, J. V.; FREI, B. Coffee and health: a review of recent human research. Critical Reviews in Food Science and Nutrition, Philadelphia, v. 46, n. 2, p. 101-123, 2006.

NICOLI, M. C.; ANESE, M.; PARPINEL, M. Antioxidants properties of coffee brews in relation to the roasting degree. LWT - Food Science and Technology, Oxford, v. 30, n. 3, p. 292-297, 1997.

NUNES, M. F.; COIMBRA, M. A. Chemical characterization of the high molecular weight material extracted with hot water from green and roasted Arabica coffee. Journal of Agricultural Food Chemistry, Washington, v. 49, n. 4, p. 1773-1782, 2001.

PARRAS, P.; MARTINÉZ-TOMÉ, M.; JIMÉNEZ, A. M.; MURCIA, M. A. Antioxidant capacity of coffees of several origins brewed following three different procedures. Food Chemistry, Oxford, v. 102, n. 3, p. 582592, 2007.

PRIOR, R. L.; WU, X.; SCHAICH, K. Standardized methods for determination of antioxidant capacity and phenolics in foods and dietary supplements. Journal of Agricultural and Food Chemistry, Washington, v. 53, n. 10, p. 4290-4302, 2005.

SÁNCHEZ-GONZALEZ，I.; JIMÉNEZ-ESCRIG，A.; SAURA-CALIXTO, F. In vitro antioxidant activity of coffees brewed using different procedures (italian, espresso and filter). Food Chemistry, Oxford, v. 90, n. 2, p. 133-139, 2005.

SHI, X.; DALAL, N. S.; JAIN, A. C. Antioxidant behavior of caffeine: efficient scavenging of hydroxyl radicals. Food and Chemical Toxicology, Brighton, v. 29, n. 1, p. 1-6, 1991.

SOUZA, R. M. N.; CANUTO, G. A. P.; DIAS, R. C. E.; BENASSI, M. T. Teores de compostos bioativos em cafés torrados e moídos comerciais. Química Nova, São Paulo, v. 33, n. 4, p. 885-890, 2010.

SWAIN, T.; HILLIS, W. E. The phenolic constituents of Prunus domestica. The quantitative analysis of phenolic constituents. Journal of the Science of Food and Agriculture, Oxford, v. 10, n. 2, p. 135-144. 1959.

TRUGO, L. C.; DE MARIA, C. A. B.; WERNECK, C. C. Simultaneous determination of total chlorogenic acid and caffeine in coffee by HPLC. Food Chemistry, Oxford, v. 42, n. 1, p. 81-87, 1991.

VIGNOLI, J. A. Influência das condições de processamento e matéria prima na composição e 
atividade antioxidante do café. 2009. Tese (Doutorado em Ciência de Alimentos) - Universidade Estadual de Londrina, Londrina.

VIGNOLI, J. A.; BASSOLI, D. G.; BENASSI, M. T. Antioxidant activity, polyphenols, caffeine and melanoidins in soluble coffee: the influence of processing conditions and raw material. Food Chemistry, Oxford, v. 124, n. 3, p. 863-868, 2011.

WEN-JEY, Y.; BOR-SEN, W.; PIN-DER, D. Antioxidant properties of roasted coffee residues. Journal of Agricultural and Food Chemistry, Washington, v. 53, n. 7, p. 2658-2663, 2005. 